\title{
Management of Persistent SARS-CoV-2 Infection in Patients with Follicular Lymphoma
}

\author{
Pilar Martínez-Barranco ${ }^{a} \quad$ María García-Roa ${ }^{a} \quad$ Roberto Trelles-Martínez ${ }^{\text {a }} \quad$ Karmele Arribalzaga $^{\text {a }}$ \\ María Velasco ${ }^{\text {b, c }}$ Carlos Guijarro $^{\text {b }}$ Javier Marcos $^{\text {b }}$ Carolina Campelo $^{d}$ \\ Juan Manuel Acedo-Sanz ${ }^{\mathrm{e}}$ Lucía Villalón ${ }^{\mathrm{a}} \quad$ Pilar Ricard $^{\mathrm{a}}$ María José García-Bueno $^{\mathrm{a}}$ \\ Elia Pérez-Fernández ${ }^{c}$ Gil Rodríguez-Caravaca ${ }^{f} \quad$ Francisco-Javier Peñalver $^{a}$ \\ ${ }^{a}$ Department of Hematology, Hospital Universitario Fundación Alcorcón, Madrid, Spain; ${ }^{b}$ Department of Internal \\ Medicine, Hospital Universitario Fundación Alcorcón, Madrid, Spain; ' ${ }^{\mathrm{R}}$ esearch Unit, Hospital Universitario \\ Fundación Alcorcón, Madrid, Spain; 'Department of Microbiology, Hospital Universitario Fundación Alcorcón, \\ Madrid, Spain; 'Department of Clinical Laboratory, Hospital Universitario Fundación Alcorcón, Madrid, Spain; \\ fDepartment of Preventive Medicine, Hospital Universitario Fundación Alcorcón, Universidad Rey Juan Carlos, \\ Madrid, Spain
}

\section{Keywords}

SARS-CoV-2 · COVID-19 · Immunocompromised patients · Follicular lymphoma · Persistent infection

\begin{abstract}
Introduction: There is no consensus on the management of the coronavirus disease (COVID-19) in patients with secondary immunosuppression due to either an underlying hematological disease or to the effects of immunochemotherapy (ICT). Some of them may present persistent infection with multiple relapses of COVID-19, requiring several admissions. This study evaluated the clinical characteristics and outcomes after treatment of 5 patients with follicular lymphoma (FL), previously treated with ICT, who developed several episodes of COVID-19. Methods: We analyzed the clinical evolution and response to treatment with antiviral agent, steroids, and convalescent plasma in 5 patients with FL and severe acute respiratory syndrome coronavirus 2 (SARS-CoV-2) persistent infection. Reverse transcriptase polymerase chain
\end{abstract}

reaction tests and peripheral blood immunophenotype were performed for all patients. Results: All patients required hospitalization due to pneumonia with severity criteria and were re-admitted after a median of 22 days (13-42) from the previous discharge. They all showed B-cell depletion by immunophenotyping, and no traces of immunoglobulin antibodies against SARS-CoV-2 were detected in any of the cases. The survival rate was $80 \%$. Conclusion: The combination therapy evidenced clinical benefits, demonstrating its capacity to control infection in immunosuppressed FL patients treated with ICT.

(c) 2021 S. Karger AG, Basel

\section{Introduction}

Severe acute respiratory syndrome coronavirus 2 (SARS-CoV-2) and its associated clinical syndrome (coronavirus disease [COVID-19]) have spread quickly around the world, becoming a major global health issue. 
Nowadays, only $14 \%$ of patients develop more severe forms of the disease, requiring oxygen therapy in hospital, and approximately $5 \%$ need intensive care unit admission [1].

Recent reports of patients re-testing positive, even after resolved symptoms and negative testing, have raised questions about the possibility of reinfection, reactivation, or persistent infection [2]. In large population-wide studies in which the age and comorbidities of patients are known, there has been suggestive evidence that patients with hematological malignancies (HM) are at increased risk of death from severe COVID-19, when compared to the general population [3]. Worse COVID-19 outcomes have been reported in hematological patients than in solid cancer and non-cancer patients [3, 4]. Furthermore, a systematic review and meta-analysis of 3,377 patients with HM and COVID-19 reported a risk of death among adults patients, most of whom were hospitalized, of $34 \%$ [5]. A population-based registry study (Madrid, Spain) of COVID-19 outcomes in patients with HM found that rates of severe/critical disease (62\%) and mortality (33\%) were between 3 and 4 times higher than those recorded in COVID-19 cases in the general population $(15 \%$ and $10 \%$, respectively) [6]. The largest series focusing specifically on COVID-19 in the lymphoma population showed that the presence of active disease was a predictor of death; however, active treatment, the number of previous lines, or type of treatment did not modify mortality risk [7]. On the other hand, a retrospective multicentric cohort study found that age $\geq 70$ years, a relapsed/refractory lymphoma, and recent administration of anti-CD20 therapy were risk factors for prolonged in-hospital stay and death for lymphoma patients hospitalized for COVID-19 [8]. Several reports described the persistence of SARSCoV-2 replication with severe symptoms for immunocompromised patients, including those with lymphoma [8-10].

We report on a case series of hematological patients who have experienced prolonged COVID-19 course with SARS-CoV-2 viremia. This study aimed to evaluate clinical characteristics and patient outcomes after treatment of persistent SARS-CoV-2 infection.

\section{Material and Methods}

\section{Samples and Patients}

This study was approved by the Ethics Committee of our center. We performed a retrospective, single-center case series of 5 patients with follicular lymphoma (FL) and persistent SARSCoV-2 infection at the University Hospital Fundación Alcorcón
(HUFA), in the Madrid area of Spain. Cases were included from March 1 to November 30, 2020 (first and second COVID-19 waves). Clinical data were obtained from electronic medical records. COVID-19 diagnosis was established based on clinical, laboratory, and imaging (X-ray or chest computed tomography [CT]) criteria. Respiratory samples and sera were collected from the patients as a part of their routine clinical care.

SARS-CoV-2 PCR: The respiratory samples were tested using the SARS-CoV-2 Viasure (Certest, Spain) using either ORF1ab and nucleocapsid or spike gene primers. Anti-SARS-CoV-2 nucleocapsid and spike immunoglobulin (IgG) were detected using a Chemiluminescent Microparticle Immuno Assay (Abbott) and automated quantitative Chemiluminescence Immuno Assay (Liaison $\mathrm{XL})$, respectively.

\section{Dosages and Definitions}

This section provides the doses used for different patient treatments and a few definitions to improve understanding of the text. Hydroxychloroquine (orally): $800 \mathrm{mg}$ for one day and $400 \mathrm{mg}$ for the next 4 days. Steroid therapy (intravenous): $6 \mathrm{mg}$ of dexamethasone, once a day for 10 days. Immunoglobulins (intravenous): 0.4 g per kilogram (kg) of weight, single dose. Remdesivir (intravenous): $200 \mathrm{mg}$ for one day and $100 \mathrm{mg}$ for next 4 days. Convalescent plasma: one unit $(300 \mathrm{cc})$ for patients under $70 \mathrm{~kg}$, and 2 units for patients over $70 \mathrm{~kg}$. Tocilizumab (intravenous): $400 \mathrm{mg}$ for patients under $75 \mathrm{~kg}$ and $600 \mathrm{mg}$ for patients over $70 \mathrm{~kg}$; single dose.

For definitions, "Day 1" was set as the day of diagnosis, after performing a reverse transcriptase polymerase chain reaction (RTPCR) test. According to the protocol, a patient was "discharged" if they had no fever for more than 2 days, as well as "clinical stability" of eupnea and comorbidities. An RT-PCR test at the time of the discharge was not available in all cases.

\section{Results}

Data from this series of patient cases are shown in Figure 1 and Table 1.

\section{Case 1}

A 47-year-old woman presented to the emergency department (ED) with fever and dry cough. A nasopharyngeal swab had tested positive for SARS-CoV-2 infection by the RT-PCR (NS-RT-PCR) assay (cycle 25). The chest $\mathrm{X}$-ray revealed bilateral pneumonia.

In 2004, the patient had been diagnosed with FL (grade 2 , stage III-A). She attained complete response (CR) after 6 cycles with fludarabine, cyclophosphamide, and rituximab (FCR), and rituximab maintenance ( $\mathrm{Rm}$ ) (last dose in September 2006). She was also diagnosed with systemic lupus erythematosus (SLE).

Treatment with antiviral agent (hydroxychloroquine) and antibiotics was started on day 1 , with progressive improvement. On day 19, the patient was discharged. 


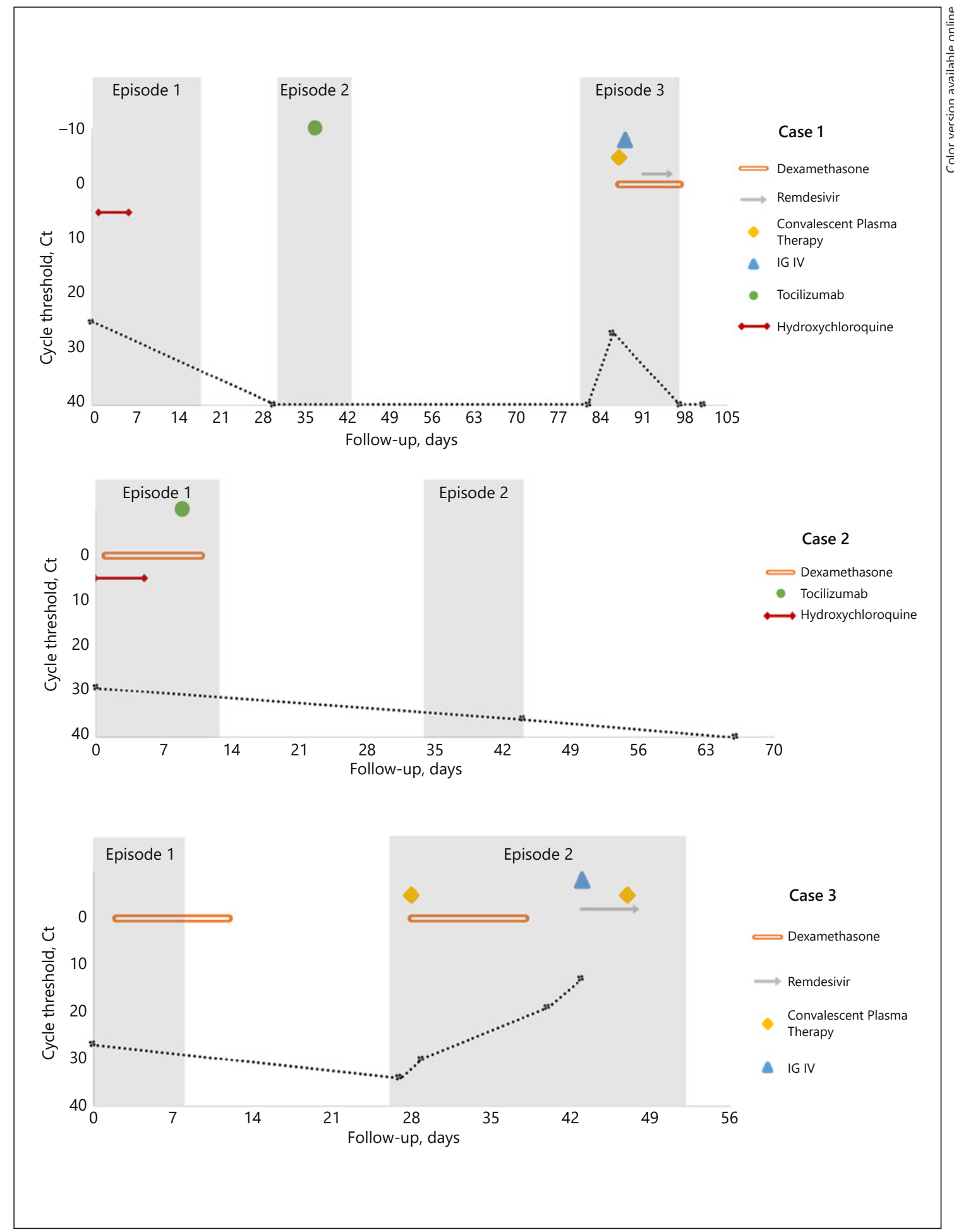

Fig. 1. Summary evolution of replicative capacity of COVID-19 (threshold cycle determined by RT-PCR) and treatments received.

(Figure continued on next page.) 


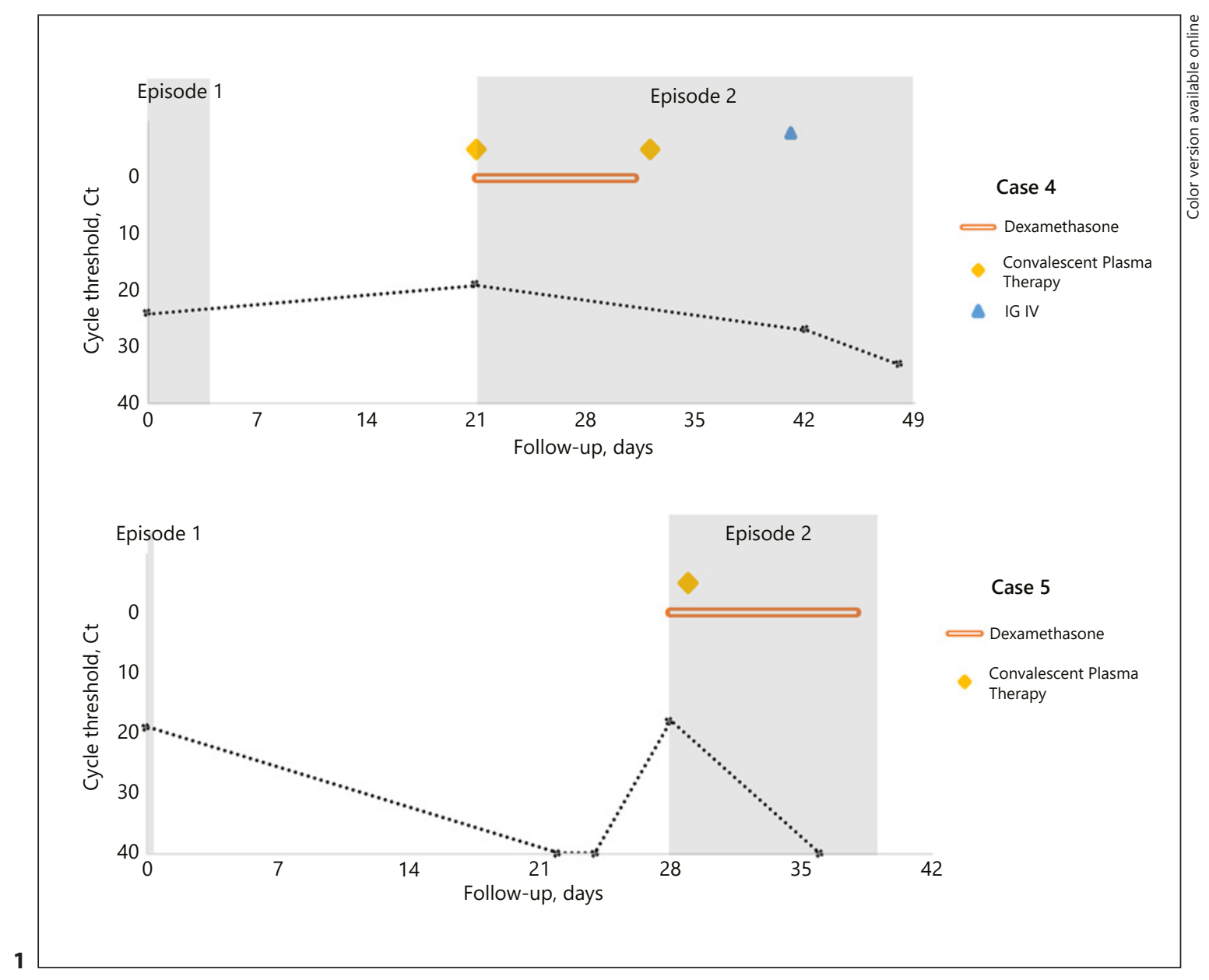

On day 31, she returned to the ED with fever and dyspnea. The chest X-ray showed radiological worsening; nevertheless, NS-RT-PCR and serologic IgG for SARS$\mathrm{CoV}-2$ tested negative. A CT was performed, identifying a viral pneumonia pattern. Any presence of another source of infection was ruled out. A severe COVID-19 episode was established based on clinical analytic-imaging criteria, which led to tocilizumab being administered on day 38. Afterward, her clinical course continued improving, and the criteria for discharge were met on day 44.

On day 83 , she was re-admitted to ED with fever and generalized arthro-myopathy. The nasopharyngeal swabs as well as serology for SARS-CoV-2 were negative. The chest X-ray and CT showed bilateral pneumonia. Other infections, reactivation of autoimmune disease, and recurrence of lymphoma were ruled out. On day 87, a bronchoscopy was performed, and a bronchoalveolar lavage (BAL) sample tested positive (cycle 30) for SARS-CoV-2.
At this time, hypogammaglobulinemia was detected, and convalescent plasma (on day 88), immunoglobulins (IGIV), steroid therapy, and remdesivir were administered as treatment. After $24 \mathrm{~h}$, the patient was afebrile, and progressive improvement in clinical and inflammatory parameters were demonstrated. NS-RT-PCR was negative on day 97. After discharge, on day 103, a new bronchoscopy was scheduled to confirm negative BAL RT-PCR (cycle 40).

\section{Case 2}

A 47-year-old man went to the ED for fever, confirming SARS-CoV-2 infection by NS-RT-PCR (cycle 29). The patient was on Rm treatment for FL (grade 2, stage IVB), attaining CR after 6 cycles of bendamustine-rituximab. $\mathrm{He}$ had received the last dose of anti-CD20 5 weeks before. A chest X-ray revealed a viral pneumonia pattern. He began treatment with hydroxychloroquine, broadspectrum antibiotic coverage, steroid therapy, and sup- 
Table 1. Baseline characteristics of patients and data for each COVID-19 episode

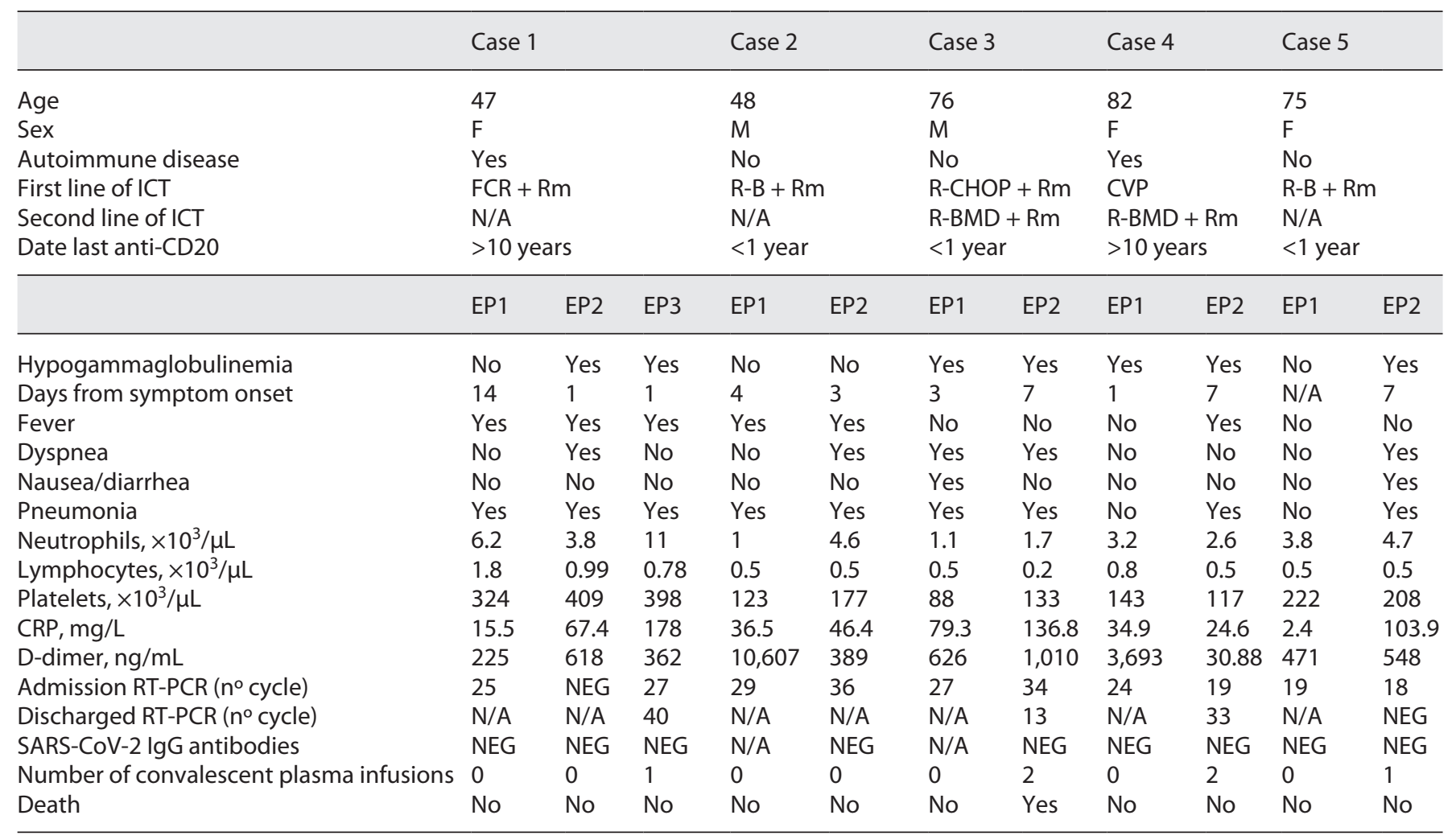

F, female; M, male; ICT, immunochemotherapy; FCR, fludarabine, cyclophosphamide, and rituximab; Rm, rituximab maintenance; R-B, bendamustine-rituximab; R-CHOP, cyclophosphamide, doxorubicin, vincristine, prednisone, and rituximab; R-BMD, rituximab, bendamustine, mitoxantrone, and dexamethasone; CVP, cyclophosphamide, vincristine, and prednisone; EP1, first episode; EP2, second episode; EP3, third episode; hypogammaglobulinemia, Ig level <500 mg/dL; N/A, not applicable; CRP, C-reactive protein; RT-PCR, reverse transcriptase polymerase chain reaction; NEG, negative.

plemental oxygen. On day 9, the patient's hypoxemia worsened, accompanied by fever and increased levels of $\mathrm{D}$-dimer. CT angiography was negative for venous thromboembolism. A dose of tocilizumab and a second pulse of systemic steroids were administered. On day 14, after $48 \mathrm{~h}$ without symptoms, the patient was discharged. On day 34, he was re-admitted after 3 days with fever and dyspnea. Bilateral pulmonary infiltrates persisted on the chest X-ray. NS-RT-PCR remained positive for SARSCoV-2 (cycle 36), whereas the serology was negative. The only treatment received during admission was antibiotic therapy. He was discharged after 5 days without symptoms (day 44). NS-RT-PCR was negative on day 66, and seroconversion for IgG COVID-19 remained negative.

\section{Case 3}

A 76-year-old man was diagnosed in 2012 with FL (grade 1, stage IV-B). He received cyclophosphamide, doxorubicin, vincristine, prednisone, and rituximab, followed by Rm for 2 years, reaching a good partial response. In 2018, progression data of FL met the criteria for further treatment. He received 4 cycles of rituximab, bendamustine, mitoxantrone, and dexamethasone, achieving CR and subsequently receiving another 2-year maintenance with rituximab (final dose received in June 2020, 16 weeks prior to entering the ED). He presented with baseline hypogammaglobulinemia.

On day 1 , the patient went to the ED with fever, dyspnea, and diarrhea. NS-RT-PCR tested positive for SARSCoV-2 (cycle 27), and chest X-ray showed bilateral pneumonia. He required oxygen and steroid therapy, which achieved a positive outcome, allowing the patient to be discharged on day 9.

On day 26, he came back to the ED due to a dry cough and progressive dyspnea. The chest X-ray was worse than before, and CT was compatible with bilateral viral pneu- 
monia. RT-PCR was positive in both nasopharyngeal swab on day 27 (cycle 34) and in BAL on day 29 (cycle 30). He received oxygen suplemment, steroid therapy, empirical broad-spectrum antibiotic, and convalescent plasma on day 28 , which led to initial improvement. Seroconversion was negative for IgG COVID-19. After 2 weeks of plasma transfusion, the clinical situation became worse upon being NS-RT-PCR positive on both day 41 (cycle 19) and day 43 (cycle 13), leading to antiviral treatment with remdesivir and a second dose of convalescent plasma on day 48. The patient died on day 53 from COVID-19 disease.

\section{Case 4}

An 82-year-old woman was diagnosed with FL (grade 1 , stage IVA), in second CR after receiving cyclophosphamide, vincristine, and prednisone in 2002 and rituximab, bendamustine, mitoxantrone, and dexamethasone followed by 2 doses of rituximab and ibritumomab tiuxetan in 2008. The last dose of anti-CD20 was administered in October 2008. She has shown baseline hypogammaglobulinemia since 2011. Currently, she receives monthly IGIV therapy. Furthermore, in 2013, she was also diagnosed with Crohn's-type inflammatory bowel disease, and subsequently provided with mesalazine and rifaximin as treatment.

She was given an asymptomatic COVID-19 diagnosis after a positive result of the SARS-CoV-2 NS-RT-PCR (cycle 24) in screening carried out after direct contact with a positive patient. On day 4 , she went to the ED with fever, but was discharged after a normal chest X-ray and appropriate breathing function. Seroconversion for IgG COVID-19 was negative.

On day 21, she came back to the ED due a high fever. She was admitted with bilateral pneumonia and a persistently positive NS-RT-PCR test (cycle 19), requiring supplemental oxygen and steroid therapy. On days 21 and 32, she received convalescent plasma transfusions. Afterward, seroconversion for IgG COVID-19 remained negative on days 29,32 , and 38 . On day 41 , she received IGIV as her usual monthly treatment for clinical immunodeficiency. She continued to show NS-RT-PCR positive on day 42 (cycle 27) and day 48 (cycle 33), but to a higher threshold cycle. Finally, the patient was discharged on day 49 after not showing symptoms for 5 days.

\section{Case 5}

A 75-year-old woman was on $\mathrm{Rm}$ treatment for FL (grade 2, stage IVA), who attained her CR after 6 cycles of immunochemotherapy (ICT) with RB. The last dose of
anti-CD20 was received 14 weeks before the diagnosis of asymptomatic SARS-CoV-2 infection by NS-RT-PCR (cycle 19). Outpatient management was performed without specific treatment, only administering full-dose anticoagulation to treat previously unknown atrial fibrillation.

On day 23, the patient went to the ED with progressive dyspnea. She was admitted with bilateral viral pneumonia, requiring supplemental oxygen. Although 2 NS-RTPCRs were negative, she tested positive in BAL on day 28 (cycle 19); however, the seroconversion IgG for SARS$\mathrm{CoV}-2$ was negative. At this time, she received steroid and empirical broad-spectrum antibiotic therapy as initial treatment. On day 30 , convalescent plasma was administered, leading to progressive improvement in her respiratory situation. The NS-RT-PCR was negative on day 36. The patient was discharged after 9 days without symptoms on day 40.

During the clinical course, all of them required hospitalization and supplemental oxygen due to pneumonia with moderate clinical severity of COVID-19 episode. None needed noninvasive ventilation or met the criteria for ICU admission. No patients developed specific antibodies against SARS-CoV-2 even after infusion of convalescent plasma. All patients followed the recommendation of home isolation and had no risk contacts. The 4 surviving patients received the vaccine against SARSCoV-2 (2 doses, AstraZeneca-Pfizer case 1, Moderna case 2, and Pfizer cases 4 and 5) between March and July 2021. Three out of 4 patients presented seroconversion +3 weeks after the second dose, detecting antibodies against SARS-CoV-2.

\section{Discussion}

We present a case series of persistent SARS-CoV-2 infection in hematological patients. All of them had been diagnosed with FL in CR before COVID-19 diagnosis. RT-PCRs of nasopharyngeal swabs or BAL samples were performed in all cases, yielding positive results. These 5 patients were re-admitted after a median of 25 days (2183 ) from the first diagnostic test, and a median of 22 days (13-42) from the previous discharge. On their second visit to the ED, they entered with recurrence of fever, viral pneumonia, and respiratory failure after initial improvement.

More data are needed to determine the specific risk factors and mechanisms that cause persistent SARSCoV-2 infection $[9,10]$. These risk factors include host 
status, virologic factors, and type and degree of immunosuppression $[1,11]$. Host factors may include sex, older age, and underlying comorbidities. The median age of our patients was 75 years (47-82), and 3 were female. Viral factors that increase the risk of persistent infection include high baseline SARS-CoV-2 load and variable genotype [11]. The RT-PCR is a common test for SARS-CoV-2, but it only detects the genetic material of the virus, without differentiating between live and dead viruses. A limitation of the test is the false-negative result, which may be attributed to a low level of viral RNA that does not reach the limit of detection [1]. In some cases, clinical and radiological worsening despite negative RT-PCR in nasopharyngeal swab indicates a shift from the upper to the lower airways, and eventually the dissemination of the virus to different body compartments: the sensitivity of BAL samples at $93 \%$, sputum samples at $72 \%$, nasal swabs at $63 \%$, and throat swabs at $32 \%[1,12]$. In our series, in 2 RT-PCR tests, patients were SARS-CoV-2 positive in the BAL samples, after having initially been negative in the nasopharyngeal swab.

Prolonged viral shedding may explain persistent infection in repeated positive SARS-CoV-2 cases. However, there is an inverse relationship between the magnitude of threshold cycle and the probability of recovering infective virus from respiratory tract samples [13]. To establish a more accurate approach, we must consider multiple factors, including the threshold cycle, the time of evolution, the clinical course, the severity of the disease, and the immunosuppression status of the patient. Although we cannot rule out reinfection by another viral strain in our patients, the temporal evolution and the absence of seroconversion suggest a persistent infection.

Patients with profound immunosuppression may shed viable SARS-CoV-2 for at least 2 months and develop persistent infection [14]. In our series, the 5 patients were all diagnosed with FL. Lymphoid malignancies such as FL may induce suppression of the immune system. In this process, neoplastic B lymphocytes can induce synapse inhibition in previously healthy $\mathrm{T}$ lymphocytes and may also promote the activity of regulatory $\mathrm{T}$ cells, contributing to further immunosuppression in the host [15]. However, all patients did achieve CR after ICT schemes. In addition to the immunosuppression produced by the lymphoid malignancy, other factors derived from the treatment could favor viral persistence. Lymphopenia is common in treated patients with bendamustine and fludarabine containing regimens as they induce prolonged $\mathrm{T}$ and $\mathrm{B}$ lymphocyte depletion. Rituximab produces sustained lymphopenia, with naive B lymphocytes and $\mathrm{CD} 4^{+} \mathrm{T}$-cell recovery at least 6 months after the last dose [16]. On the other hand, associated to B-cell depletion, rituximab produces hypogammaglobulinemia. This alteration of both humoral and cellular immunity translates into an increased risk of infection, including viral infections $[15,17]$. Moreover, secondary hypogammaglobulinemia after ICT may result in an adverse event for patients; however, data on this finding are sparse. In an Israeli study, which mostly included relapsed or refractory patients with non-Hodgkin lymphoma who were treated with BR, baseline hypogammaglobulinemia (IgG $<500 \mathrm{mg} / \mathrm{dL}$ ) was found in $19.7 \%$ of patients. The accumulated incidence of hypogammaglobulinemia at the end of follow-up was $39.5 \%$ for the entire group and $27.8 \%$ for patients without hypogammaglobulinemia at baseline $[15,18]$.

In accordance with these observations, 4 of 5 of the patients in this study showed hypogammaglobulinemia at baseline or during clinical course. Memory B cells are crucial for the development of immunity against SARSCoV-2 [19]. No SARS-CoV-2-specific antibodies were detectable prior to the treatment with convalescent plasma in any of the patients. Peripheral blood immunophenotyping was performed in all cases, showing B-cell depletion and an inversion of the CD4/CD 8 ratio, which could indicate a T-reactive process against viruses. However, the patients remained unable to control SARSCoV-2 viremia despite a broad T-cell response. Three of the patients developed COVID-19 during maintenance with rituximab at 2, 5, and 16 weeks after the last dose of anti-CD20; as such, lymphopenia with B and T depletion may contribute to the persistence of the virus and the inability to develop antibodies. In a large-scale case series of COVID-19 in patients with HM, the authors reported that patients receiving monoclonal antibody-based therapy had a significantly greater (HR 2.02) risk of death than those not receiving active antineoplastic treatment [6]. The 2 remaining patients received the last dose of rituximab more than 10 years ago, which did not justify the lymphodepletion state by FL or ICT. But both patients have immune system dysfunction: case 1 developed an autoimmune disease (SLE) and case 4 had long-standing symptomatic severe hypogammaglobulinemia, being treated with support IgG, and subsequently developed inflammatory bowel disease.

Regarding treatments for COVID-19 overall, few strategies have provided benefits; several have failed, and others are still being evaluated. Therefore, we decided to treat these 5 patients using combination therapy with dexamethasone, remdesivir, and convalescent plasma. The ra- 
tionale for using this combination therapy was to achieve sustained virological response. Thus far, only 2 agents have shown a degree of clinical efficacy in large randomized, controlled trials: remdesivir, in hospitalized patients with pulmonary disease, and dexamethasone, in hospitalized patients receiving oxygen [20,21]. Remdesivir suppresses the viral replication [22], whereas convalescent plasma could provide neutralizing antibodies for patients with lymphopenia and hypogammaglobulinemia, who cannot produce specific antibodies themselves.

Plasma infusions have an adequate safety profile and have been associated with improved outcomes in patients who have had other infectious diseases [23, 24]. However, the PlasmArtrial has reported that the use of convalescent plasma did not result in a significant clinical benefit, as compared to placebo in patients with severe COVID-19 pneumonia [23]. Nevertheless, in different randomized clinical trials, the use of convalescent plasma in the general population with COVID-19 pneumonia requiring hospitalization did not offer a statistically significant benefit in terms of overall survival $[25,26]$. However, convalescent plasma infusion must be administered soon after infection in order to be effective, although the most appropriate antibody concentrations for effective treatment remain unclear. A recently published randomized clinical trial shows that the administration of high-titer convalescent plasma to infected older adults within $72 \mathrm{~h}$ after the onset of mild symptoms reduced the progression of $\mathrm{CO}$ VID-19 to severe illness [24]. Moreover, a retrospective multicenter cohort study, real-world data, included 44,720 patients hospitalized with COVID-19 demonstrated that convalescent plasma is significantly associated with reduced risk on in-hospital mortality [27]. Additionally, there is a large matched control retrospective study which found that convalescent plasma therapy was associated with a survival benefit in patients with HM [28]. Consistent with our findings, a study conduted by Bertrains et al. [29] showed that protracted COVID-19 in patients with B-cell lymphoma is associated with a lack of significant neutralizing antibody titers and impaired clearance of SARS-CoV-2 therapy. Patients with several forms of immunosuppression would be good candidates for passive transfer of immunity by COVID-19 convalescent plasma therapy $[29,30]$. Another therapeutic option, a neutralizing antibody combined cocktail REGN-COV2 (casirivimab and imdevimab), against SARS-CoV-2 can also be an effective antiviral therapy, enhancing viral clearance and thus leading to improved outcomes, particularly in patients whose own immune response to the virus is slow to initiate [31].

Management of Persistent SARS-CoV-2 Infection
Limitations of the present study include its retrospective design, small sample size, and data from a single center. Furthermore, we could not rule out a new infection in our patients by another strain, since we do not have access to these viral cultures nor genome sequencing.

\section{Conclusion}

In this study, immunosuppression produced by lymphoma or secondary to treatment with ICT and maintenance with rituximab are associated with an increased risk of developing persistent SARS-CoV-2 infection. After administering our treatment strategy, 4 of 5 patients in our series are alive, sustaining CR of their hematological disease as well as a resolved SARS-CoV-2 infection. The management of these patients is not defined. In our experience, the use of treatment combined with the antiviral agent, dexamethasone (only if respiratory support is required), and convalescent plasma early is capable of controlling SARS-CoV-2 infections and resulting in positive patient outcomes.

\section{Acknowledgements}

We wish to thank all of the patients and their families who participated in this study, as well as all of the healthcare staff involved in the management of hematological patients with COVID-19.

\section{Statement of Ethics}

This study was approved by Institutional Review Boards (NoCEIm 20/182) and the local Ethical Committee (20/85). All procedures followed were in accordance with the ethical standards of the responsible committee on human experimentation (institutional and national) and with the Helsinki Declaration of 1975 , as revised in 2008. Institutional Review Boards granted, given the emergency situation of the disease and that it is an observational, retrospective study and without intervention, with a relevant scientific interest, the inclusion of patients will be allowed without signature of the informed consent, which itself should be reflected in the verbal consent in the clinical history and even ratification when possible, all with authorization from the CEIC, as defined in SAS Order 3470/2009 and related clarifying documents issued by the AEMPS (Agencia Española de Medicamentos y Productos Sanitarios).

\section{Conflict of Interest Statement}

The authors declare no conflicts of interest. 


\section{Funding Sources}

This study received no funding from external sources.

\section{Author Contributions}

P. Martínez-Barranco conceived of the presented idea and wrote the first draft. P. Martínez-Barranco, M. García-Roa, and R. Trelles-Martínez developed the theory and collected data. P. Martínez-Barranco, M. García-Roa, R. Trelles-Martínez, and F.J. Peñalver revised the intermediate and final draft of manuscript. All authors carefully revised and fully approved the final manuscript. P. Martínez-Barranco, M. García-Roa, R Trelles-Martínez, F.J. Peñalver, K. Arribalzaga, M. Velasco, C. Guijarro, J. Marcos, L. Villalón, P. Ricard, and M.J. García-Bueno treated patients and col- lected data; C. Campelo provided assistance with sample collection, testing, and RT-PCR analyses; J.M. Acedo-Sanz provided assistance with sample collection and testing SARS-CoV-2-specific antibodies analyses; P. Martínez-Barranco and E. Pérez-Fernández were responsible for the analysis and interpretation of the data; and G. Rodríguez-Caravaca critically revised the manuscript.

\section{Data Availability Statement}

The research data are not available for legal and ethical reasons, due to the Data Protection Law in force in Spain. However, if any reviewer requires any additional information, it can be requested from the corresponding author or the Institutional Review Board (IRB), under reasonable justification.E-mail address: hufa.ceim@ salud.madrid.org.

\section{References}

1 Osman AA, Al Daajani MM, Alsahafi AJ. Repositive coronavirus disease 2019 PCR test: Could it be a reinfection? New Microbes New Infect. 2020;37:100748.

2 Duggan NM, Ludy SM, Shannon BC, Reisner AT, Wilcox SR. Is novel coronavirus 2019 reinfection possible? Interpreting dynamic SARS-CoV-2 test results through a case report. Am J Emerg Med. 2021 Jan;39:256.e1-3.

3 El-Sharkawi D, Iyengar S. Haematological cancers and the risk of severe COVID-19: exploration and critical evaluation of the evidence to date. Br J Haematol. 2020;190(3):336-45.

4 Cattaneo C, Daffini R, Pagani C, Salvetti M, Mancini V, Borlenghi E, et al. Clinical characteristics and risk factors for mortality in hematologic patients affected by COVID-19. Cancer. 2020;126(23):5069-76.

5 Vijenthira A, Gong IY, Fox TA, Booth S, Cook G, Fattizzo B, et al. Outcomes of patients with hematologic malignancies and COVID-19: a systematic review and meta-analysis of 3377 patients. Blood. 2020;136(25):2881-92.

6 García-Suárez J, De la Cruz J, Cedillo A, Llamas $\mathrm{P}$, Duarte R, Jiménez-Yuste V, et al. Asociación madrileña de hematología y hemoterapia (AMHH). Impact of hematologic malignancy and type of cancer therapy on COVID-19 severity and mortality: lessons from a large population-based registry study. J Hematol Oncol. 2020;13(1):133.

7 Regalado-Artamendi I, Jiménez-Ubieto A, Hernández-Rivas JA, Navarro B, Nuñéz L, Alaez C, et al. Risk factors and mortality of COVID-19 in Patients With Lymphoma: A Multicenter Study. HemaSphere. 2021 Feb 10; 5(3):e538.

8 Duléry R, Lamure S, Delord M, Di Blasi R, Chaulet A, Hueso T, et al. Prolonged in-hospital stay and higher mortality after COVID-19 among patients with non-Hodgkin lymphoma treated with B-cell depleting immunotherapy. Am J Haematol. 2021 Aug 1; 96(8):934-44.
9 Nakajima Y, Ogai A, Furukawa KJ, Arai R, Anan R, Nakano Y, et al. Prolonged viral shedding of SARS-CoV-2 in an immunocompromised patient. J Infect Chemother. 2021; 27(2):387-9.

10 Reuken PA, Stallmach A, Pletz MW, Brandt C, Andreas N, Hahnfeld S, et al. Severe clinical relapse in an immunocompromised host with persistent SARS-CoV-2 infection. Leukemia. 2021;35(3):920-3.

11 Ye G, Pan Z, Pan Y, Deng Q, Chen L, Li J, et al. Clinical characteristics of severe acute respiratory syndrome coronavirus 2 reactivation. J Infect. 2020;80(5):e14-e17.

12 Wang W, Xu Y, Gao R, Lu R, Han K, Wu G, et al. Detection of SARS-CoV-2 in different types of clinical specimens. JAMA. 2020;323:1843-4.

13 García F, Melón S, Navarro D, Paño JR, Galán JC. Organización del diagnóstico de SARSCoV-2 y estrategias de optimización. Sociedad Española de Enfermedades Infecciosas y Microbiología Clínica; 2020. p. 1-14.

14 Ayllo T, Gonzalez-Reiche AD, Aslam S, Van de Guchte A, Khan Z, Obla A, et al. Shedding of viable SARS-CoV-2 after Immunosuppressive therapy for cancer. N Engl J Med. 2020; 383(26):2586-8.

15 Gafter-Gvili A, Polliack A. Bendamustine associated immune suppression and infections during therapy of hematological malignancies. Leuk Lymphoma. 2016;57(3):512-9.

16 Yasuda H, Tsukune Y, Watanabe N, Sugimoto K, Uchimura A, Tateyama M, et al. Persistent COVID-19 pneumonia and failure to develop Anti-SARS-CoV-2 antibodies during rituximab maintenance therapy for follicular lymphoma. Clin Lymphoma Myeloma Leuk. 2020;20(11):774-6.

17 Lukenbill J, Kalaycio M. Fludarabine: a review of the clear benefits and potential harms. Leuk Res. 2013;37(9):986-94.
18 Gafter-Gvili A, Ribakovsky E, Mizrahi N, Avigdor A, Aviv A, Vidal L, et al. Infections associated with bendamustine containing regimens in hematological patients: a retrospective multi-center study. Leuk Lymphoma. 2015;56:1-7.

19 Gaebler C, Wang Z, Lorenzi JCC, Muecksch F, Finkin S, Tokuyama M, et al. Evolution of antibody immunity to SARS-CoV-2. Nature. 2021;591(7851):639-44.

20 Beigel JH, Tomashek KM, Dodd LE, Mehta AK, Zingman BS, Kalil AC, et al. Remdesivir for the treatment of COVID-19: final report. N Engl J Med. 2020;383(19):1813-26.

21 Horby P, Lim WS, Emberson JR, Mafham M, Bell JL, Linsell L, et al. Dexamethasone in hospitalized patients with COVID-19. N Engl J Med. 2021;384(8):693-704.

22 Camprubí D, Gaya A, Marcos MA, Martí-Soler H, Soriano A, Mosquea MDM, et al. Persistent replication of SARS-CoV-2 in a severely immunocompromised patient treated with several courses of remdesivir. Int J Infect Dis. 2021;104:379-81.

23 Simonovich VA, Burgos Pratx LD, Scibona P, Beruto MV, Vallone MG, Vázquez C, et al. A randomized trial of convalescent plasma in COVID-19 severe pneumonia. N Engl J Med. 2021;384(7):619-29.

24 Libester R, Pérez Marc G, Wappner D, Coviello S, Bianchi A, Braem V, et al. Early hightiter plasma therapy to prevent severe COVID-19 in older adults. N Engl J Med. 2021; 384(7):610-8.

25 Avendaño-Solá C, Ramos-Martínez A, Muñez-Rubio E, Ruiz-Antorán B, Malo de Molina $\mathrm{R}$, Torres $\mathrm{F}$, et al. A multicenter randomized open-label clinical trial for convalescent plasma in patients hospitalized with $\mathrm{CO}$ VID-19 pneumonia. J Clin Invest. 2021 Oct 15;131(20):e152740. 
26 Körper S, Weiss M, Zickler D, Wiesmann T, Zacharowski K, Corman VM, et al. Results of the CAPSID randomized trial for high-dose convalescent plasma in severe COVID-19 patients. J Clin Invest. 2021 Oct 15;131(20): e152264.

27 Arnold Egloff SA, Junglen A, Sa Restivo J, Wongskhaluang M, Martin C, Doshi P, et al. Convalescent plasma associates with reduced mortality and improved clinical trajectory in patients hospitalized with COVID-19. J Clin Invest. 2021 Oct 15;131(20):e151788.
28 Thompson MA, Henderson JP, Shah PK, Rubinstein SM, Joyner MJ, Choueiri TK, et al. Association of convalescent plasma therapy with survival in patients with hematologic cancers and COVID-19. JAMA Oncol. 2021 Jun 17;7(8):1167-75.

29 Betrains A, Godinas L, SheridaWoei-A-Jin HFJ, Rosseels W, Van Herck Y, Lorent N, et al. Convalescent plasma treatment of persistent severe acute respiratory syndrome coronavirus-2 (SARS-CoV-2) infection in patients with lymphoma with impaired humoral immunity and lack of neutralising antibodies. $\mathrm{Br}$ J Haematol. 2021 Mar;192(6):1100-5.
30 Senefeld JW, Klassen SA, Ford SK, Senese KA, Wiggins CC, Bostrom BC, et al. Use of convalescent plasma in COVID-19 patients with immunosuppression. Transfusion. 2021 Aug; 61(8):2503-11.

31 Weinreich DM, Sivapalasingam S, Norton T, Ali S, Gao H, Bhore R, et al. REGN-COV2, a neutralizing antibody cocktail, in outpatients with COVID-19. N Engl J Med. 2021;384: $238-51$. 